\title{
BMJ Open COpenhagen Neuroplastic TRaining Against Contractures in Toddlers (CONTRACT): protocol of an open- label randomised clinical trial with blinded assessment for prevention of contractures in infants with high risk of cerebral palsy
}

Maria Willerslev-Olsen (10 ,1,2 Jakob Lorentzen, ${ }^{1}$ Katrine Røhder, ${ }^{3}$ Anina Ritterband-Rosenbaum, ${ }^{1,4}$ Mikkel Justiniano, ${ }^{1,4}$ Andrea Guzzetta, ${ }^{5}$ Ane Vibeke Lando, ${ }^{6}$ Anne-Mette Bæk Jensen, ${ }^{6}$ Gorm Greisen, ${ }^{6}$ Sofie Ejlersen, ${ }^{2}$ Line Zacho Pedersen, ${ }^{2}$ Britta Andersen, ${ }^{2}$ Patricia Lipthay Behrend, ${ }^{2}$ Jens Bo Nielsen ${ }^{1}$

To cite: Willerslev-Olsen $M$, Lorentzen J, Røhder K, et al. COpenhagen Neuroplastic TRaining Against Contractures in Toddlers (CONTRACT): protocol of an open-label randomised clinical trial with blinded assessment for prevention of contractures in infants with high risk of cerebral palsy. BMJ Open 2021;11:e044674. doi:10.1136/ bmjopen-2020-044674

- Prepublication history for this paper is available online. To view these files, please visit the journal online (http://dx.doi org/10.1136/bmjopen-2020044674).

Received 09 September 2020 Accepted 02 June 2021

Check for updates

(C) Author(s) (or their employer(s)) 2021. Re-use permitted under CC BY-NC. No commercial re-use. See rights and permissions. Published by BMJ.

For numbered affiliations see end of article.

Correspondence to Dr Maria Willerslev-Olsen; mwo@elsassfonden.dk

\section{ABSTRACT}

Introduction Contractures are frequent causes of reduced mobility in children with cerebral palsy (CP) already at the age of 2-3 years. Reduced muscle use and muscle growth have been suggested as key factors in the development of contractures, suggesting that effective early prevention may have to involve stimuli that can facilitate muscle growth before the age of 1 year. The present study protocol was developed to assess the effectiveness of an early multicomponent intervention, CONTRACT, involving familyoriented and supervised home-based training, diet and electrical muscle stimulation directed at facilitating muscle growth and thus reduce the risk of contractures in children at high risk of CP compared with standard care.

Methods and analysis A two-group, parallel, open-label randomised clinical trial with blinded assessment $(n=50)$ will be conducted. Infants diagnosed with CP or designated at high risk of $\mathrm{CP}$ based on abnormal neuroimaging or absent fidgety movement determined as part of General Movement Assessment, age 9-17 weeks corrected age (CA) will be recruited. A balanced 1:1 randomisation will be made by a computer. The intervention will last for 6 months aiming to support parents in providing daily individualised, goal-directed activities and primarily in lower legs that may stimulate their child to move more and increase muscle growth. Guidance and education of the parents regarding the nutritional benefits of docosahexaenic acid (DHA) and vitamin $\mathrm{D}$ for the developing brain and muscle growth will be provided. Infants will receive DHA drops as nutritional supplements and neuromuscular stimulation to facilitate muscle growth. The control group will receive standard care as offered by their local hospital or community. Outcome measures will be taken at $9,12,18$, 24, 36 and 48 months CA. Primary and secondary outcome measure will be lower leg muscle volume and stiffness

\section{Strengths and limitations of this study}

- This study is the first randomised controlled clinical trial with blinded assessment to investigate the effect of an early multicomponent intervention involving family oriented and supervised home-based training, diet and electrical muscle stimulation directed at facilitating muscle growth and thus reduce the risk of contractures in children at high risk of cerebral palsy compared with standard care.

- Validated objective techniques will be used for primary and secondary outcome measures.

- The study is unique in covering a longitudinal followup until the age of 4 years.

- The intervention is a combination between several different components that makes it difficult to decide which of the different components is the most significant; thus, the effect achieved will be a synergistic effect of this combination.

of the triceps surae musculotendinous unit together with infant motor profile, respectively.

Ethics and dissemination Full approval from the local ethics committee, Danish Committee System on Health Research Ethics, Region H (H-19041562). Experimental procedures conform with the Declaration of Helsinki.

Trial registration number NCT04250454.

Expected recruitment period 1 January 2021-1 January 2025.

\section{INTRODUCTION}

Cerebral palsy (CP) is the most common cause of motor and cognitive disabilities 
in childhood affecting around 1 out of 500 infants. ${ }^{1-3}$ Contractures and joint deformities are frequent complications, which limit the functional abilities of the children from an early age and are a main life-long challenge for social integration and participation. ${ }^{4-7}$ The functional limitations imposed by the combination of weak paretic muscles and contractures in early childhood also reduce the child's possibilities of active exploration of the environment and social interaction with peers. ${ }^{8-10}$ This may have secondary impact on cognitive development and cognitive performance later in life. ${ }^{1112}$

Therefore, early intervention to prevent contractures is pivotal in helping motor and cognitive development in children with $\mathrm{CP}$ and thereby enable social integration and optimal cognitive and motor performance throughout their lifetime. The importance of prevention of contractures is also emphasised by the realisation that none of the surgical, medical or physical therapies that are available provide an efficient treatment of contractures once they have become manifest and interfere with joint mobility. ${ }^{13-15}$

There is growing evidence that reduced growth of muscles is a key factor in the development of contractures. ${ }^{16-20}$ This relates both to length and diameter of muscle fibres, since increased diameter of muscle fibres has been found to account for a large part of the growth in length of muscles. ${ }^{1621}$ If muscles fail to grow at the same rate as bones, they will be subjected to abnormal tension. ${ }^{1620}$ This and the lack of muscle use may stimulate growth of connective tissue in the muscles causing a stiffer extracellular matrix. ${ }^{22}$ Growth of the medial gastrocnemius (MG) muscle in infants with CP deviates from that of typically developing (TD) infants around the age of 12-15 months. ${ }^{1723}$ Pathologically increased stiffness of the muscle tissue is seen some months later consistent with a causal relation between reduced growth and increased stiffness. ${ }^{1724}$ These findings indicate that efficient prevention of contractures will have to take place before the age of 1 year and will need to focus on stimulation of muscle growth as a key factor.

Muscle growth depends crucially on muscle use. ${ }^{25}$ How do we get a child who has difficulty activating its muscles to do so when that child has no prior experience of using its muscles and limbs and has little verbal understanding of the goal of intervention?

It has been shown that reduced muscle growth precedes the development of muscle stiffness in children with CP already before the age of 2 years. ${ }^{17}$ Thus, in order to confirm the outcome of an early intervention, longitudinal follow-up will be essential. As for the measurement of increased stiffness, quantification has been shown to have varying reproducibility and reliability when assessed without objective equipment. Several recent studies have further pointed out that the necessity of distinguishing different contributions to muscle stiffness in clinical setting is crucial. ${ }^{172627}$ Hence, to assess the impact of the intervention, the use of an objective method will be critical.
There is now evidence to support that the early development of the motor system is highly plastic and depends crucially on activity-dependent interaction with the environment. ${ }^{2-35}$ Experimental evidence from kittens and rodents supports the existence of a sensitive period soon after birth where descending connections from the motor cortex to the spinal cord are reorganised in an activity-dependent manner. ${ }^{28-35}$ Functional deficits in mature animals who have received central motor lesions prior to or in relation to birth appear to depend on the extent to which this activity-dependent reorganisation takes place during the sensitive period. It is unknown whether a similar sensitive period exists in humans, but there is reason to believe that the first 5-6months after birth constitute a period where the motor system undergo rapid changes that may resemble the sensitive period in rodents and kittens. This period is characterised in humans by so-called fidgety movements (FMs), which may reflect a 'calibration' of the sensorimotor system, where descending connections are reorganised similar to what is seen in animals. ${ }^{36}$ Children also acquire the ability of goal-directed movements using visual feedback during this period, which may be related to maturation of the connections from motor cortex to the spinal cord. ${ }^{38}$ There is also evidence that children aged 5-6 months have acquired a sense of agency over their own movements and a basic understanding of how they may use their body to control their environment. ${ }^{39}$ Sensory feedback and reward that are associated to successful behaviour play an important role in this early establishment of movement control. ${ }^{4-42}$ Thelen and Fisher ${ }^{43}$ showed that when infants at a very early age can activate a mobile by own spontaneous movements, movement of the infant increased. The initial 5-6months after term may therefore provide a window of opportunity where it is possible to facilitate neurodevelopmental reorganisation, which enhances movement and thereby may attenuate contractures through the concomitant muscle growth stimulus. An intervention in which infants in that age group are stimulated to move by their parents at home under supervision by therapists ${ }^{44}$ has indeed been demonstrated to improve motor development in infants with high risk of $\mathrm{CP}^{45}$ Home-training technology that may facilitate the training and the interaction between therapists and the families are also now becoming available and have shown promising effects on motor development in preterm infants. ${ }^{46} 47$ Intensive goal-oriented training involving experiences of success and frequent reward within the first 5-6months is therefore central in the intervention that we propose in the present protocol for prevention of contractures in infants at high risk of developing CP.

Muscle growth does depend on neural and mechanical stimuli and on nutritional and metabolic stimuli. It is therefore important also to consider the nutritional status of the infant and especially whether nutrients that have a specific muscle growth promoting effect are delivered in sufficient quantity to the infant either through breastfeeding or breast milk substitutes. The 
polyunsaturated fatty acid, docosahexaenic acid (DHA) is considered essential for maturation of the developing brain $^{48}$ and may also facilitate muscle growth. ${ }^{49}$ Supplementation with DHA is not recommended for healthy term-born infants ${ }^{50}$ but may be important for development of preterm infants and especially for infants with brain lesion. ${ }^{5152}$ In addition, vitamin $\mathrm{D}$ also plays a role in regulating muscle growth, ${ }^{53} 54$ and deficiency of vitamin $\mathrm{D}$ has been suggested to be of importance in neurodevelopmental disorders. ${ }^{55}$ Information about the nutritional status of mother during pregnancy and mother and infant postbirth could therefore be of importance to enhance facilitation of muscle growth in the infants.

It should also be considered whether stimuli that may substitute the neural activation of the muscles such as electrical stimulation may be used when the child is not otherwise active (for instance during sleep) to help maintain muscle growth. Electrical muscle stimulation has been shown to diminish muscle atrophy in adults. ${ }^{5657}$ The facilitation of muscle growth may help to postpone contracture development until communication with the (older) infant is easier and training goals may therefore be achieved more easily.

The purpose of the present paper is consequently to describe a study protocol for a single blinded randomised controlled clinical trial of an early multicomponent intervention programme directed towards facilitating muscle growth and thus reduce the risk of contractures in the lower leg in children at high risk of CP compared with standard care. ${ }^{26}$ This is a multicomponent intervention involving family oriented and supervised home-based training, diet and electrical muscle stimulation to maximise efficacy and clinical applicability.

\section{Objective}

The aim of this study is to evaluate whether a multicomponent individual intervention, initiated within 9-17 weeks corrected age (CA) and directed at facilitating muscle growth may reduce the risk of contractures in children with $\mathrm{CP}$ more than standard care.

\section{METHODS AND ANALYSIS}

A two-group open-label randomised clinical trial with blinded assessment $(n=36)$ will be applied to compare COpenhagen Neuroplastic TRaining Against Contractures in Toddlers (CONTRACT) with standard care (see paragraph further).

All outcome measures for both CONTRACT and control will be assessed at baseline, postintervention and at $9,12,18,24,36$ and 48 months CA. In order to characterise all infants included, all participants will be assessed at baseline, before randomisation, with a neurological examination (Hammersmith Infant Neurological Examination), The Alberta Infant Motor Scale, Bayley III and parents will be asked to fill out the questionnaire Ages \& Stages (ASQ-3).
The primary and secondary outcomes of this trial are the volume of the MG muscle ${ }^{23}$ and the stiffness of the triceps surae musculotendinous unit of the infants ${ }^{24} 26$ as well as the infant motor profile (IMP) ${ }^{58}$ respectively. Both will be measured before and after intervention and at 9, 12, 18, 24, 36 and 48 months CA (IMP will only be used from 3 to 18 months CA).

We hypothesise that:

1. Infants diagnosed with $\mathrm{CP}$ or at risk of $\mathrm{CP}$ that receive CONTRACT intervention will have a larger increase in MG muscle volume compared with infants receiving standard care.

2. Infants diagnosed with $\mathrm{CP}$ or at risk of $\mathrm{CP}$ that receive CONTRACT intervention will have a smaller increase in the stiffness of the triceps surae musculotendinous unit and a higher IMP score than infants receiving standard care.

\section{Patient and public involvement}

No patient and public involvement.

\section{Ethics and dissemination}

Written and verbal information will be given to the parents or primary caregiver at least 48 hours before consent will be obtained. All parents/primary caregiver will be asked to provide written consent prior to participation.

The local ethics committee, Region $\mathrm{H}$, has granted approval of the study (H-19041562). Experimental procedures conform with the Declaration of Helsinki.

\section{Recruitment}

Fifty infants will be recruited from neonatal and paediatrics departments in Denmark. All parents of eligible infants will be informed about the study only after they have had consultations with their medical team regarding the high-risk status of their child, or a confirmed diagnosis of CP. Families will be given a site-specific information sheet regarding the purpose and design of the study and have opportunity to speak with the investigators before consenting to the study. After consent is obtained, prior to randomisation, the family will be invited to visit the Elsass Foundation for 2 days to complete all baseline assessments and collect demographic and perinatal data. All families, whether in the intervention or control group, will be offered a consultation with an experienced psychologist during their stay (figure 1.). In case the parents need actual psychological treatment due to mental health or other severe difficulties that cannot be handled within the team, efforts will be made to ensure that they are offered treatment by proper specialists.

MRI and medical data will be obtained from the infant's medical record.

\section{Inclusion criteria}

Infants younger than 17 weeks CA with suspicion of brain lesion determined from a medical assessment, by MRI or ultrasound (US) scans or absence of FMs as determined by General Movement Assessment will be included. The brain lesion should be rated severe enough by the 


\section{Eligible infants ( $<15$ weeks $C A)$;}

Brain lesion determined from MRI or ultrasound scanning or absent fidgety movements.

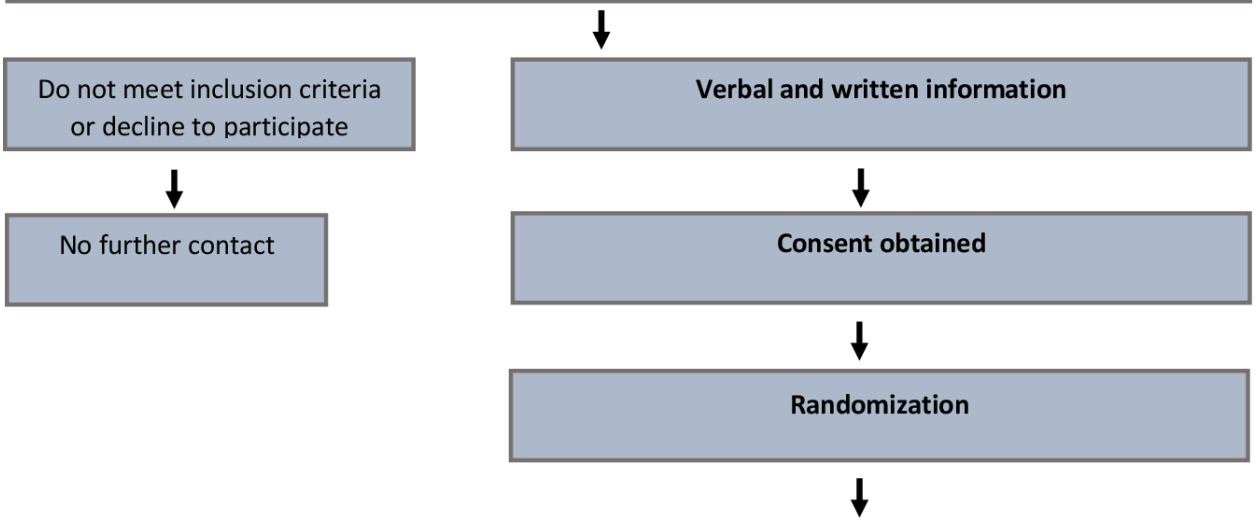

2-3 days with baseline measures and psychological conversation for all recruited.

Primary: Muscle volume (rate of muscle growth) and evaluation of stiffness of the triceps surae musculotendinous unit.

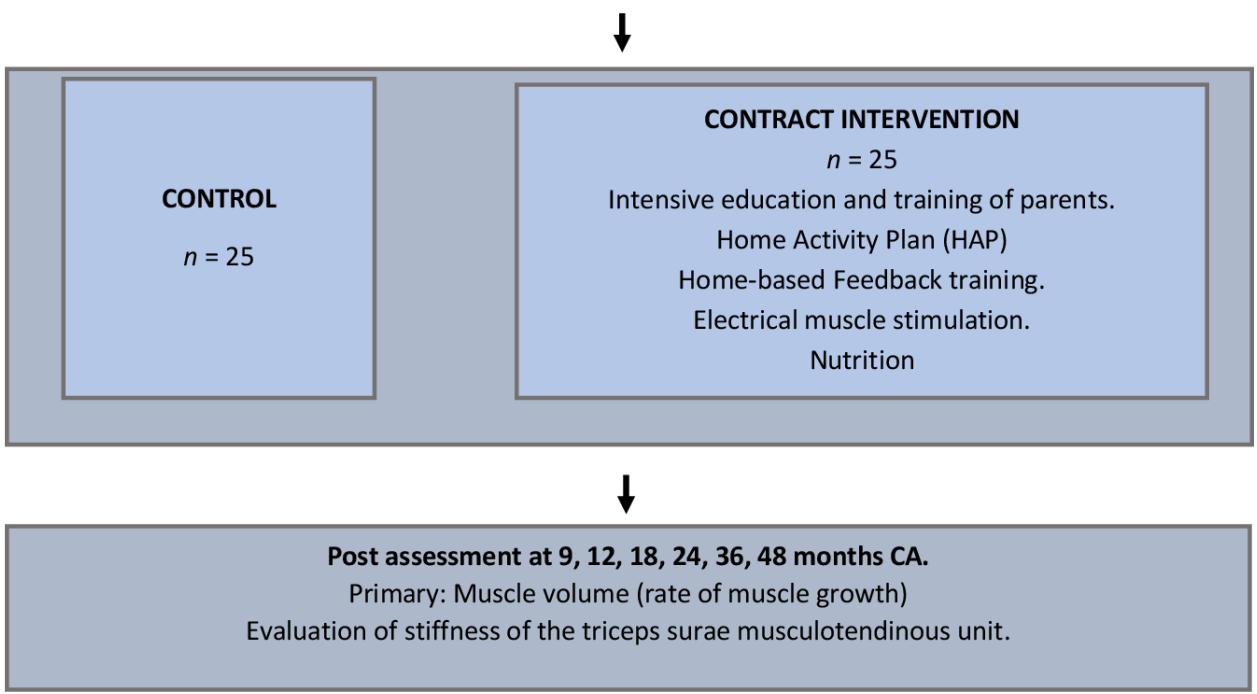

Figure 1 Flow chart. CA, corrected age.

clinician to have informed the parents of the associated risk of CP. Absence of FM has been shown to be highly predictive for CP especially in combination with MRI $\left(95 \%-98 \%{ }^{59}\right)$.

MRI and medical data will be obtained from the infant's medical record.

\section{Exclusion criteria}

Infants otherwise eligible but with severe genetic abnormalities, severe heart problems, metabolic diseases or still hospitalised will not be selected for the study.

\section{Sample size}

Currently, there is little knowledge of the effect of early intervention on muscle growth; thus, estimation is based on data from a previous study where the muscle growth for TD children and children with CP was attained. ${ }^{17}$
Planned sample size is based on power calculation using growth rates of $0.52 \mathrm{~mL} /$ month for TD children and $0.28 \mathrm{~mL} /$ month for children with $\mathrm{CP}^{17}$ Data from a previous pilot study demonstrated an increase in muscle growth of $6.2 \mathrm{~mL}$ after 3 months of intervention $(1.7 \mathrm{~mL} /$ month) in children with CP. ${ }^{60} \mathrm{We}$, thus, find it reasonable to expect that the intervention will cause an increase in muscle growth of $0.24 \mathrm{~mL} /$ month. With an SD of 0.23 , a power of $80 \%$ and an alpha value of 0.01 , sample size is estimated to $n=42$ (21 per group).

Four children were added to each group to take possible drop out into account ( $\mathrm{n}=50,25$ in each group).

\section{Randomisation process}

When informed consent has been obtained, participants will be randomised using computer-generated balanced 
randomisation. Children will be stratified based on age, gender and side of the lesion (left/right).

In case of twins, these will be randomised together due to the nature of the intervention.

\section{Blinding process}

Both families, the treating therapist and investigators will be blinded to group allocation until after baseline testing. Due to the nature of the intervention, it is not possible for either the participating families or the investigators conducting the intervention to be blinded.

All data sampling and analysis will be performed by investigators, unaware of group allocation. Final analysis of data will be performed consecutively for each infant following baseline and endpoint measurements and locked until final statistical analysis at the end of the study.

\section{Safety and adverse events}

To monitor adverse events, all parents will be questioned by the principal investigator every second week, and all adverse events will be documented. All adverse events, regardless of severity, will be documented and reported. If serious, the ethics committee will be notified, and the child's treating physician will be informed. Prior to participating, risk assessments including strategies to avoid and minimise will be completed.

\section{CONTRACT intervention}

CONTRACT is an acronym for COpenhagen Neuroplastic TRaining Against Contractures in Toddlers.

The team in charge of CONTRACT consists of medical doctors, exercise physiologists, physiotherapists, occupational therapists and psychologists with specific expertise in infant development. This multidisciplinary approach is intended to ensure that it will be possible to make a coordinated approach that target as many challenges for the child and the family as possible.

All members of the team will have received specific training and education in early diagnosis and intervention based on contemporary motor knowledge.

The intervention will last for 6 months, starting when the infant is around 15 weeks $\mathrm{CA}$ and consist of five elements:

1. Intensive training and education of parents (2-3 days).

2. Home activity plan (HAP).

3. Home-based feedback training.

4. Electrical muscle stimulation.

5. Nutrition supplementation.

Combination of these elements has been chosen in order to ensure that optimal muscle growth is achieved through multimodal stimulation of the motor and cognitive development of the child.

\section{Personal meeting and detailed information}

All parents included in the intervention group will be invited to the Elsass Foundation to a personal meeting and detailed presentation about the development of their infant. Current knowledge of infants' development and neuroplasticity will be introduced to the parents.
The parents will receive guidance and inspiration about how to stimulate/play with their infant as one important element of the HAP (see next section for further details). This includes knowledge about how reading and interpreting the signals of the infant can be made in a way that is based on current knowledge on infant development and neuroplasticity. The latter will be individualised based on the results of baseline assessments and clinical history of the infant.

The decisive impact of a balanced energy level and the importance of nutritional and metabolic stimuli in order to optimise muscle growth in the infant will be emphasised. Knowledge concerning why DHA and vitamin D are essential for the developing brain and muscles and thus may be important for the development of infants will be presented. The nutritional status of mother and infant regarding nutrition supplements will be monitored. In case of identified eating difficulties such as chewing, swallowing, reflux, etc, and oral exercises and therapeutic assistance will be provided. All participants, infants as well as mothers, will furthermore be encouraged to follow the Danish recommendations regarding nutritional supplementation. Questionnaires will ensure gathering of important information regarding breast feeding, milk formulas and additional supplementation (eg, lactic bacteria) of the infant. If difficulties or special needs are observed, a therapist with expertise in this area will be consulted.

\section{Home activity plan}

The purpose of the HAP is to help parents and/or other primary caregivers to stimulate the cognitive and motor development of the infant with special focus on the use of lower limb muscles as much as possible through daily play activities. The HAP provides: (1) general guidelines and principles for the play activities based on current knowledge of neuroplasticity and motor learning ${ }^{4561}$ (this will take place during the 'Personal meeting and detailed information' for the parents during the stay) as well as (2) inspirational examples of play activities tailored to the functional abilities of the individual infant.

\section{General guidelines and principles}

Parents will receive extensive information about the general guidelines and principles for the HAP prior to the intervention. Continued compliance with the principles will be ensured through supervision by specially trained therapists every second week (personal consultation for 1-3 hours once a month and video consultation 1-2 hours once a month with 2 weeks in between). The following principles will be emphasised:

\section{High intensity and long duration}

To ensure sufficient intensity of the intervention, the play activities will be provided by the parents and/or other primary caregivers in the home of the child as part of daily activities (changing diaper, eating and playing). The activities will be performed daily including weekends in the 
full intervention period (180 days in total (6 months)). The training should last $30-45$ min every day and may be performed consecutively or separately in blocs when the infant is alert and compliant. At the end of each activity session, the parents register the performed activity by placing an ' $x$ ' on a pretyped list of activity categories and an ' $\mathrm{x}$ ' for duration $(2,5,10,20,30$ and $40 \mathrm{~min})$.

\section{Individualisation and progression}

All activities should be adjusted to the functional abilities of the infant and should be made more challenging as the infant acquires new abilities. The parents will be instructed about this during the initial education where therapists will help to adjust the activities according to the initial abilities of the infant. Further adjustments and progression will be facilitated through videoconferences and emails between therapists and parents every 2 weeks throughout the intervention period. Furthermore, the catalogue of examples of play activities will be organised according to different functional abilities of infants (see further).

\section{Active participation}

To ensure that the infant is alert and takes active part, the activities should be performed when the infant is fed and rested. Eye contact and/or attention is essential. Parents should always aim to organise the play activities in a way that require motor and cognitive responses from the infant. Based on the initial cues made by the parent, the child should thereby be facilitated to make movements of the limbs or repositioning of the body. The stimuli given by the parents could be verbal, tactile or visual but always with the aim of initiating responses from the infant.

\section{Motivation and reward}

Parents will be coached to notice and identify things that create a joyful reaction from their child and use these to motivate the child to perform the activities and/or as reward when an activity has been performed successfully. The need of providing the reward immediately and as a clear recognition of the successful achievement of the activity will be emphasised. The parents will also be instructed to gradually increase the requirements for reward when the child shows progression of abilities.

\section{Play activities}

The activities will be chosen/inspired from a library of play activities that will be presented to the parents (see table 1).

One to two days of training of parents and/or other primary caregivers in the practical performance of the activities will be provided by specially educated therapists prior to the intervention. This will take place in continuation with the initial stay or in separate days according to the need of the families.

The play activities are all designed to induce muscle activity in the lower limbs of the infant and should be seen as examples of activities rather than a specific manual for activities. The activities can be performed in different settings such as on the floor, in a stroller, in bed or outside on the lawn and can be modified according to the preferences of the parents and infant. The play activities will be chosen according to the capabilities of the infant and will be progressed as the infant acquires new capabilities. Examples of activities for three positions of the infant (supine position, prone position and supported standing) including suggestions for performance, progression, active infant involvement, motivation and intensity are described in table 1 .

\section{Home-based feedback training}

Prediction and particularly development of prediction is to a large extent dependent on motor activity, and in order to understand the action of others, it is crucial to understand your own motor repertoire. ${ }^{62}$ Feedback training has been shown to have a positive influence on motor activity. A former experiment showed that when infants at a very early age could activate a mobile by the movement of their own spontaneous leg kicks, the kick rate increased. ${ }^{43}$ The basic idea is that the infants' movements generate a positive reinforced sensory feedback that will then promote more movements. This was also demonstrated in a study where infants were given the opportunity to view their own movements via a realtime video recording (Rochat and Morgan 1995). ${ }^{63}$ This illustrated that feedback-generated training is feasible in infants at the age of 2-3 months and thus that infants have the capacity to link sensory information with their own motor output. ${ }^{42}$

Based on experience from previous work on home-based intervention technologies, ${ }^{64}$ a user-friendly computerbased feedback system has been developed. This system will be given to the family for a minimum of 3-4 days a week (preferably daily) training of approximately $20 \mathrm{~min}$ (which can be divided into shorter training sessions depending on the participation of the infant) during all of the intervention period. The system consists of an application running on a smartphone/tablet and a set of four wireless movement sensors (Movesense, Suunto) attached to each of the infant's extremities. The system analyses the infant's movements, which are then used as input to control visual and auditory stimuli presented to the infant. The stimuli are presented through the app on the device, held in the field of view of the infant, using a dedicated mechanical tablet arm. These stimuli are only introduced when the infant's motor activity exceed a given threshold. Adaptive filtering is applied to the movement signal to change the activity threshold according to the infant's level of movement, thus requiring minimal influence from the parents. The movement data and stimuli activation are logged on the device for evaluating the progress of the infant. To respect privacy and to follow the General Data Protection Regulation (GDPR) legislation, the logged data do not include video data from the camera but only the raw data from the movement sensors, as well as information of the presented stimuli. 


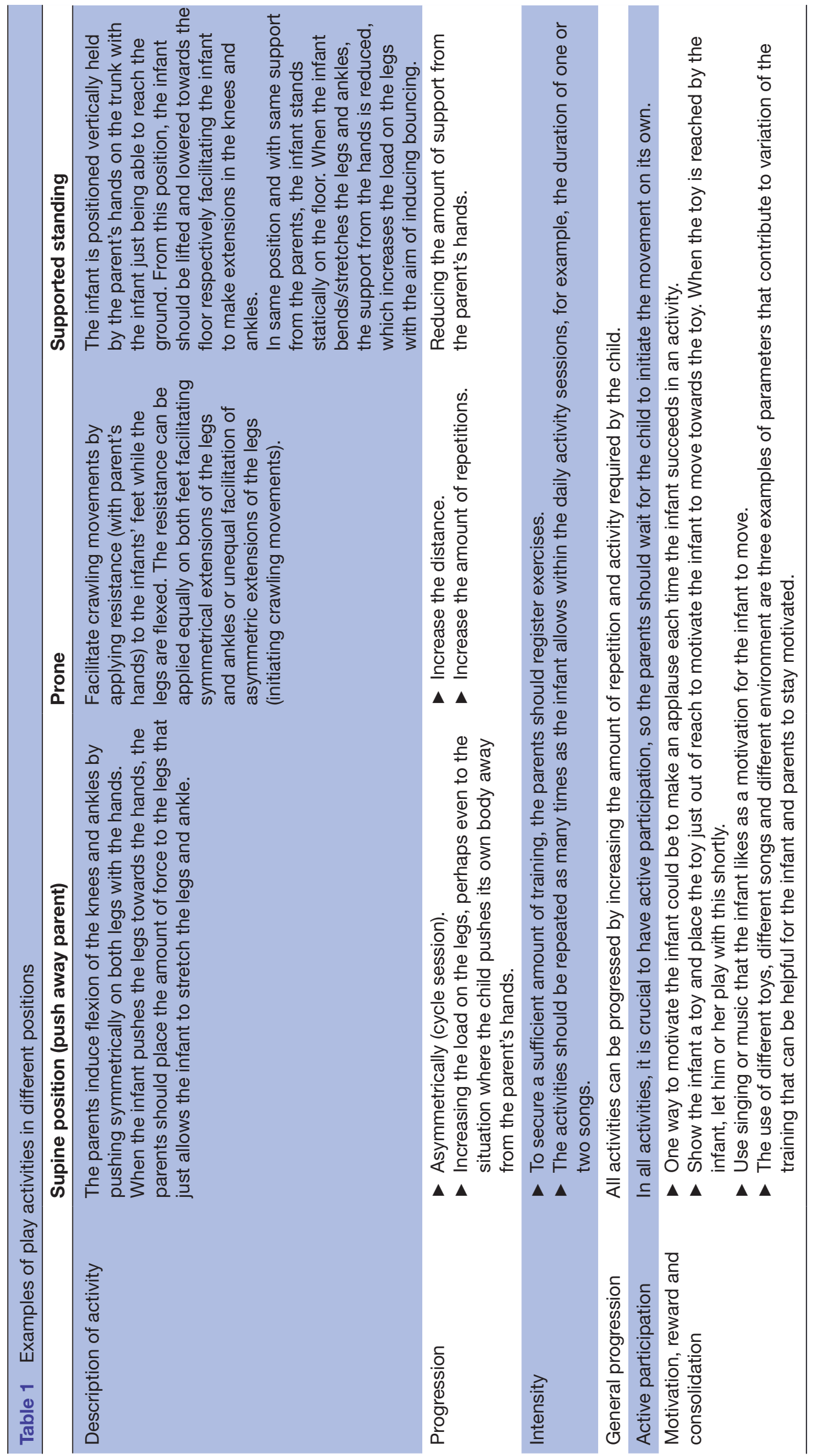




\section{Electrical muscle stimulation}

All infants will receive $25 \mathrm{~min}$ of daily electrical stimulation of the triceps surae muscle group bilaterally during sleep or during playing at an intensity sufficient to cause muscle contraction (Complex muscle stimulator Sport 4.0, programme: rehabilitation muscle atrophy). During the stay at the Elsass Foundation, after randomisation, parents will be introduced to the use of the stimulation and have the opportunity to use the equipment with their infant. The frequency and intensity of the stimulation will be documented throughout the intervention. Electrical stimulation has been shown to facilitate muscle growth in older children with $\mathrm{CP}^{65}$ but has to our knowledge not been tested in this age group before.

\section{Nutrition}

It is ensured that all participants, infants as well as mothers follow the Danish recommendations regarding nutritional supplementation. Moreover, all infants (from 4 months of age) will receive daily DHA (Nordic Naturals Omega-3 Mini, https://www.webapoteket.dk/helbred/ kost-og-helse/nordic-naturals-omega-3-mini-p-222068, $38 \mathrm{mg} / \mathrm{kg}$ body weight) and vitamin D supplementation (10 $\mu \mathrm{g}$ according to the Danish recommendations).

Questionnaires will be used to obtain information regarding breast feeding, milk formulas and additional supplementation (eg, lactic bacteria) of the infant. In addition, evaluation of oral motor skills will be performed on all infants. If difficulties or special needs are observed a therapist with expertise in this area will be associated.

\section{Standard care}

Standard care is the follow-up as well as the therapeutic interventions offered by the hospital and/or community when an infant is considered to be at high risk of CP. All children included will be offered standard care by their local hospital or community. The approach used as well as the frequency of the interventions are varied and not possible to standardise. All families included in the control group will be offered individual consultation with a psychologist from the Elsass Foundation in the same way as for the intervention group. Furthermore, participation in specially organised classes for families with children with CP will be offered from the age of 12 months by the Elsass Foundation.

Frequency and type of therapeutic intervention, for example, physiotherapy, occupational therapy, oral therapy or psychologist as well as orthoses, botulinium toxin A or oral medication, during the 6 months of intervention will be noted for each family.

\section{Outcome measures}

Primary outcome measure

Muscle volume

US measurement of the muscle volume is evaluated at each test session using a computer-based B-mode US scanner with a 128 -element beam former and a $10 \mathrm{MHz}$ linear transducer with a $60 \mathrm{~mm}$ field of view (Echoblaster
128, Telemed, Vilnius, Lithuania). The volume of the MG muscle is calculated using Stradwin software. Muscle volume reflects growth in both length and diameter of muscle fibres, which both contribute to muscle length. Measures of muscle volume have been validated in infants with $\mathrm{CP}$, and clinically meaningful changes in growth have been defined.

Researchers with high experience in the use of US examine the entire length of the MG muscle to assess muscle volume, muscle belly length and muscle composition. Height, weight, circumference of the widest part of the crus and fibula length are measured. US is performed on the most affected leg or if possible, on both legs, with the infant in a prone position and ankle in a relaxed position. To estimate muscle thickness and fascicle length, one recording is performed with the probe positioned longitudinally at the midbelly of the MG, with the infant's ankle fixed in a $90^{\circ}$ angle. The probe is handheld and placed vertically with the lower leg for all images.

A computer-based B-mode US scanner with a 128element beam former and a $10 \mathrm{MHz}$ linear transducer with a $60 \mathrm{~mm}$ field of view (Echoblaster 128; Telemed) is used to record US images. Freehand three-dimensional US is used to measure muscle volume as has been previously described and validated. ${ }^{667}$ The volume of the MG muscle is calculated using Stradwin software. Morphological parameters will be normalised to the length of fibula to account for growth versus intervention. US measurements will be carried out prior to intervention, postintervention and at 9, 12, 18, 24, 36 and 48 months CA.

For further details, see refs 1723.

\section{Secondary outcome measure}

\section{Passive stiffness and reflex stiffness}

Passive and reflex mediated stiffness of the ankle plantar flexors will be objectively assessed according to the methods described in Lorentzen et $a l^{27}$ and WillerslevOlsen et al. ${ }^{17}$ With the subject in supine position and with the use of a PSAD device (a handheld medical device, https://movotecdevices.com/), ${ }^{26}$ the researcher will first move the ankle joint from maximal plantar flexed position to maximal dorsiflexion in order for the device to estimate the range of movements (ROMs).

To estimate the passive stiffness of the ankle plantar flexors, manual movements will be performed by the researcher at a slow velocity $(\sim<20 \mathrm{deg} / \mathrm{s})$ through the entire ROM 5-10 times to make sure that a minimum of three excellent trials will be collected. Only trials in which no electromyography (EMG) activity is observed in the plantar flexor muscles is accepted for further analysis.

With the purpose of investigating the stretch reflex onset and stretch reflex-mediated stiffness, 5-10 trials at a velocity as fast as possible, will be performed.

Data will be sampled at a rate of $512 \mathrm{~Hz}$ and transferred to a computer via Bluetooth for further analysis in Matlab (Mathworks, Natick, Massachusetts, USA). EMG activity is sampled from bipolar surface EMG electrodes $(0.5 \mathrm{~mm}$ diameter, $2 \mathrm{~cm}$ between electrodes; Ambu Blue Sensor 
NF-00-S/12; Ambu, Ballerup, Denmark) placed over the plantar flexor muscles at the and the dorsal flexor muscle. The device is equipped with strain gauges, which permit measurement of the applied torque during the slow and fast stretches. Stiffness will be calculated at any given point of the movement as ratio between the torque and the movement. The evaluation will be carried out prior to intervention, postintervention, 6 months postintervention as well as every 12 months until the age of 51 months.

\section{Analysis and statistics}

All data will be managed and analysed using the Statistical Package for Social Sciences (SPSS 28). Descriptive statistics (means, SD) will be calculated to summarise the data set for both groups and to identify potential baseline differences between the groups; $p$ values will be used to indicate the strength of the evidence. The primary level of analysis will be the assessment of the effects of training on muscle volume (primary outcome measure) and agerelated change in muscle stiffness (secondary outcome measure). Linear mixed models (fixed effect analysis) (covariance parameter best model fit) will be used to test for interaction effects between the intervention group and control group before and after the 6 months of training/control period and 3 months after training. Post hoc test for differences between measurements before intervention, after the 6 months intervention period and 3 months after, and differences between the measurements of the control group will be made using the Sidak method.

Secondary outcome measures will be used for exploratory data analysis. In the second-level analysis, we will provide multivariate statistics in order to take into account the effect of the basal level of motor development, nutritional status, family compliance and time of training. It is anticipated that some families will say no to electrical muscle stimulation and that there will be high variability in the time and intensity of stimulation. This will allow for a subset analysis to address the influence of electrical stimulation separately for the different outcome measures.

The analyses will be made in IBM SPSS V.22 statistics.

\section{DISCUSSION}

This paper describes a single blinded RCT protocol that compares a novel intervention 'CONTRACT' with standard care to prevent development of contractures in infants at high risk of CP. There is general consensus that there is a need of controlled clinical trials that document the efficiency of early interventions in infants at risk of neurodevelopmental disorders such as $\mathrm{CP}^{68-70} \mathrm{We}$ have chosen to focus on muscle growth and muscle stiffness, since these may be measured by well-documented objective techniques that have been validated in several studies on the target population of infants. ${ }^{176667}$ There is furthermore increasing evidence that muscle growth and muscle stiffness are important factors in the pathophysiology of contractures and joint deformities in children with CP. ${ }^{16-20} 22$ Contractures and joint deformities are in turn major limitations for development of functional abilities, mobility and participation. ${ }^{4-7}$ We have decided to make measurements at 6-12 months intervals until the infants are 51 months since studies have shown that muscle growth and muscle stiffness in CP infants deviate significantly from TD infants in this time period. This should provide more sensitive and less error prone outcome measures than what will be achieved when relying on a single measure at a given time point after the intervention.

We have chosen to combine several different components in the intervention (parental guidance and support in home-based training, feedback training, electrical stimulation and nutrition), although this makes it difficult to decide which of the different components is efficient. However, as pointed out in the Introduction, there are good reasons to assume that all components will have some effects on muscle growth and muscle stiffness and that synergistic effects will be achieved by combining them. We believe that it is realistic by a maximally intensive intervention to achieve near-normal muscle growth rates in the intervention group, while the control group, due to the high level of predictive power of the combination of brain imaging and general movement analysis, will follow a growth rate near that of infants in whom CP will subsequently be diagnosed. In this decision, it was also considered important to ensure a sufficiently large effect of the intervention to keep the number of infants included in the study as low as possible. CP is relatively rare, and a study in which a larger number of infants would need to be recruited would not be feasible. Finally, the significance of the individual components for the observed effects may possibly be evaluated from variations in compliance as a post hoc analysis.

In the case that this study will provide the proof of principle, then clinical value must be studied in larger phase III trials.

\section{Ethics and dissemination}

Full approval from the local ethics committee, Danish Committee System on Health Research Ethics, Region H (Journal no. H-19041562). Experimental procedures conform with the Declaration of Helsinki.

\section{Trial registration}

ClinicalTrials.gov. Date 29 January 2020.

The protocol follows the recommendations of the Consolidated Standards of Reporting Trials statement 2010.

In the case of potential changes concerning the protocol, an additional application will be done and sent for approval in the ethics committee.

Results will be disseminated in peer-reviewed publications and presentations at national and international conferences. 


\section{Consent for publication}

All authors have read and accepted publication of the manuscript.

\section{Availability of data and materials \\ Not applicable.}

\section{Study status}

Recruitment of participation has not started.

\section{Confidentiality}

All necessary personal information will be anonymised, stored and saved according to the GDPR.

Furthermore, all processing of personal data will be in accordance with the directions of the University of Copenhagen. Consent will be obtained prior to participation, and it will always be possible to withdraw a signed consent without further question. Consent is stored securely.

\section{Author affiliations}

${ }^{1}$ Department of Neuroscience, University of Copenhagen Faculty of Health Sciences, Copenhagen, Denmark

${ }^{2}$ Department of Research, Elsass Fonden, Charlottenlund, Denmark

${ }^{3}$ Department of Psychology, Unversity of Copenhagen, Copenhagen, Denmark

${ }^{4}$ Elsass Foundation, Charlottenlund, Denmark

${ }^{5}$ Department of Neurology, Stella Maris Institute, Pisa, Italy

${ }^{6}$ Neonatatal Department, Rigshospitalet, Kobenhavn, Denmark

Acknowledgements We would like to thank the Elsass Foundation for the financial support throughout the planning and for thepossibility to initiate the project.

Contributors MW-0: conceived and designed the protocol and wrote first draft of the manuscript, approved the final version of the manuscript and will contribute during the study with education of parents or primary caregiver, testing and analysing data as well as preparing and write the manuscript based on the study. $\mathrm{JL}$ : conceived and designed the protocol, edited and revised the manuscript, approved the final version of the manuscript and will contribute during the study with education of parents or primary caregiver, testing and analysing data as well as preparing and write the manuscript based on the study. KR: edited and revised the manuscript, approved the final version of the manuscript, will contribute during the study with education of parents or primary caregiver as well as both testing and analysing data amd responsible for the psychological assessment of the parents. AR-R: edited and revised the manuscript, approved the final version of the manuscript and will contribute during the study with both testing and analysing data. MJ: edited and revised the manuscript, approved the final version of the manuscript and will contribute during the study with as technical asistance and analysing data. AG: edited and revised the manuscript and approved the final version of the manuscript. AVL, A-MBJ and GG: edited and revised the manuscript, approved the final version of the manuscript and will be repsponsible for the recruitement during the study. SE and LZP: edited and revised the manuscript, approved the final version of the manuscript and will contribute during the study with education of parents or primary caregiver as well as both testing and analysing data. BA: edited and revised the manuscript, pproved the final version of the manuscript and will contribute during the study with education of parents or primary caregiver as well as both testing and analysing data. PLB: edited and revised the manuscript, approved the final version of the manuscript and will supervise the overall progress of the study. JBN: conceived and designed the protocol, edited and revised the manuscript, approved the final version of the manuscript and will supervise the research, analysing data as well as preparing and write the manuscript based on the study.

Funding The project is financially supported by The Elsass Foundation. The funding covers salaries for MW-0, AR-R, MJ, SE, LZP, BA and PLB, 90\% salary for JBN and $50 \%$ for JL and KR. No external peer review has taken place regarding the funding. Possible changes concerning the protocol will not influence the funding.

Competing interests None declared.

Patient and public involvement Patients and/or the public were not involved in the design, or conduct, or reporting, or dissemination plans of this research.
Patient consent for publication Not required.

Provenance and peer review Not commissioned; externally peer reviewed.

Open access This is an open access article distributed in accordance with the Creative Commons Attribution Non Commercial (CC BY-NC 4.0) license, which permits others to distribute, remix, adapt, build upon this work non-commercially, and license their derivative works on different terms, provided the original work is properly cited, appropriate credit is given, any changes made indicated, and the use is non-commercial. See: http://creativecommons.org/licenses/by-nc/4.0/.

ORCID iD

Maria Willerslev-Olsen http://orcid.org/0000-0001-7817-6610

\section{REFERENCES}

1 Rosenbaum P. The natural history of gross motor development in children with cerebral palsy aged 1 to 15 years. Dev Med Child Neurol 2007;49:724.

2 Ravn SH, Flachs EM, Uldall P. Cerebral palsy in eastern Denmark: declining birth prevalence but increasing numbers of unilateral cerebral palsy in birth year period 1986-1998. Eur J Paediatr Neurol 2010;14:214-8

3 Graham HK, Rosenbaum P, Paneth N, et al. Cerebral palsy. Nat Rev Dis Primers 2016;2:15082.

4 Chiu H-C, Ada L, Butler J, et al. Relative contribution of motor impairments to limitations in activity and restrictions in participation in adults with hemiplegic cerebral palsy. Clin Rehabil 2010;24:454-62.

5 Murphy KP. The adult with cerebral palsy. Orthop Clin North Am 2010;41:595-605.

6 Horstmann HM, Hosalkar H, Keenan MA. Orthopaedic issues in the musculoskeletal care of adults with cerebral palsy. Dev Med Child Neurol 2009;51 Suppl 4:99-105.

7 Hägglund G, Wagner P. Spasticity of the gastrosoleus muscle is related to the development of reduced passive dorsiflexion of the ankle in children with cerebral palsy: a Registry analysis of 2,796 examinations in 355 children. Acta Orthop 2011;82:744-8.

8 Uldall P. Everyday life and social consequences of cerebral palsy. Handb Clin Neurol 2013;111:203-7.

9 Shikako-Thomas K, Majnemer A, Law M, et al. Determinants of participation in leisure activities in children and youth with cerebral palsy: systematic review. Phys Occup Ther Pediatr 2008;28:155-69.

10 Fox AS, Carty CP, Modenese L, et al. Simulating the effect of muscle weakness and contracture on neuromuscular control of normal gait in children. Gait Posture 2018;61:169-75.

11 Spittle A, Orton J, Anderson PJ, et al. Early developmental intervention programmes provided post hospital discharge to prevent motor and cognitive impairment in preterm infants. Cochrane Database Syst Rev 2015;11:CD005495.

12 Oudgenoeg-Paz O, Mulder $\mathrm{H}$, Jongmans MJ, et al. The link between motor and cognitive development in children born preterm and/ or with low birth weight: a review of current evidence. Neurosci Biobehav Rev 2017;80:382-93.

13 Tedroff K, Granath F, Forssberg H, et al. Long-Term effects of botulinum toxin A in children with cerebral palsy. Dev Med Child Neurol 2009;51:120-7.

14 Tedroff K, Löwing K, Jacobson DNO, et al. Does loss of spasticity matter? A 10-year follow-up after selective dorsal rhizotomy in cerebral palsy. Dev Med Child Neurol 2011;53:724-9.

15 Wallen M, Stewart K. The evidence for abandoning upper limb stretch interventions in paediatric practice. Dev Med Child Neurol 2013;55:208-9.

16 Gough M, Shortland AP. Could muscle deformity in children with spastic cerebral palsy be related to an impairment of muscle growth and altered adaptation? Dev Med Child Neurol 2012;54:495-9.

17 Willerslev-Olsen M, Choe Lund M, Lorentzen J, et al. Impaired muscle growth precedes development of increased stiffness of the triceps surae musculotendinous unit in children with cerebral palsy. Dev Med Child Neurol 2018;60:672-9.

18 Barber L, Barrett R, Lichtwark G. Passive muscle mechanical properties of the medial gastrocnemius in young adults with spastic cerebral palsy. J Biomech 2011;44:2496-500.

19 Barber L, Hastings-Ison T, Baker R, et al. Medial gastrocnemius muscle volume and fascicle length in children aged 2 to 5 years with cerebral palsy. Dev Med Child Neurol 2011;53:543-8.

20 Gough M. Muscle deformity in cerebral palsy: reduced use, overuse, or both? Dev Med Child Neurol 2009;51:768-9.

21 McNee AE, Gough M, Morrissey MC, et al. Increases in muscle volume after plantarflexor strength training in children with spastic cerebral palsy. Dev Med Child Neurol 2009;51:429-35. 
22 Smith LR, Lee KS, Ward SR, et al. Hamstring contractures in children with spastic cerebral palsy result from a stiffer extracellular matrix and increased in vivo sarcomere length. J Physiol 2011;589:2625-39.

23 Herskind A, Ritterband-Rosenbaum A, Willerslev-Olsen M, et al. Muscle growth is reduced in 15-month-old children with cerebral palsy. Dev Med Child Neurol 2016;58:485-91.

24 Willerslev-Olsen M, Lorentzen J, Sinkjaer T, et al. Passive muscle properties are altered in children with cerebral palsy before the age of 3 years and are difficult to distinguish clinically from spasticity. Dev Med Child Neurol 2013;55:617-23.

25 Counts BR, Buckner SL, Mouser JG, et al. Muscle growth: to infinity and beyond? Muscle Nerve 2017;56:1022-30.

26 Yamaguchi T, Hvass Petersen T, Kirk H, et al. Spasticity in adults with cerebral palsy and multiple sclerosis measured by objective clinically applicable technique. Clin Neurophysiol 2018;129:2010-21.

27 Lorentzen J, Grey MJ, Crone C, et al. Distinguishing active from passive components of ankle plantar flexor stiffness in stroke, spinal cord injury and multiple sclerosis. Clin Neurophysiol 2010;121:1939-51.

28 Chakrabarty S, Friel KM, Martin JH. Activity-Dependent plasticity improves M1 motor representation and corticospinal tract connectivity. J Neurophysiol 2009;101:1283-93.

29 Martin JH, Chakrabarty S, Friel KM. Harnessing activity-dependent plasticity to repair the damaged corticospinal tract in an animal model of cerebral palsy. Dev Med Child Neurol 2011;53 Suppl 4:9-13.

30 Williams PTJA, Jiang Y-Q, Martin JH. Motor system plasticity after unilateral injury in the developing brain. Dev Med Child Neurol 2017;59:1224-9.

31 Clowry GJ, Walker L, Davies P. The effects of botulinum neurotoxin A induced muscle paresis during a critical period upon muscle and spinal cord development in the rat. Exp Neurol 2006;202:456-69.

32 Gibson CL, Arnott GA, Clowry GJ. Plasticity in the rat spinal cord seen in response to lesions to the motor cortex during development but not to lesions in maturity. Exp Neurol 2000;166:422-34.

33 Martin JH, Donarummo L, Hacking A. Impairments in prehension produced by early postnatal sensory motor cortex activity blockade. J Neurophysiol 2000;83:895-906.

34 Martin JH, Friel KM, Salimi I, et al. Activity- and use-dependent plasticity of the developing corticospinal system. Neurosci Biobehav Rev 2007;31:1125-35.

35 Clowry GJ. The dependence of spinal cord development on corticospinal input and its significance in understanding and treating spastic cerebral palsy. Neurosci Biobehav Rev 2007;31:1114-24.

36 Guzzetta A, Mercuri E, Rapisardi G, et al. General movements detect early signs of hemiplegia in term infants with neonatal cerebral infarction. Neuropediatrics 2003;34:61-6.

37 Hadders-Algra M. Putative neural substrate of normal and abnormal General movements. Neurosci Biobehav Rev 2007;31:1181-90.

38 Wallace PS, Whishaw IQ. Independent digit movements and precision grip patterns in 1-5-month-old human infants: handbabbling, including vacuous then self-directed hand and digit movements, precedes targeted reaching. Neuropsychologia 2003;41:1912-8.

39 Wang Q, Bolhuis J, Rothkopf CA, et al. Infants in control: rapid anticipation of action outcomes in a gaze-contingent paradigm. PLoS One 2012;7:e30884.

40 Sargent B, Kubo M, Fetters L. Infant discovery learning and lower extremity coordination: influence of prematurity. Phys Occup Ther Pediatr 2018;38:210-25.

41 Sargent B, Schweighofer N, Kubo M, et al. Infant exploratory learning: influence on leg joint coordination. PLoS One 2014;9:e91500.

42 Ritterband-Rosenbaum A, Justiniano MD, Nielsen JB, et al. Are sensorimotor experiences the key for successful early intervention in infants with congenital brain lesion? Infant Behav Dev 2019;54:133-9.

43 Thelen E, Fisher DM. From spontaneous to instrumental behavior: kinematic analysis of movement changes during very early learning. Child Dev 1983;54:129-40.

44 Morgan C, Novak I, Dale RC, et al. GAME (Goals - Activity - Motor Enrichment): protocol of a single blind randomised controlled trial of motor training, parent education and environmental enrichment for infants at high risk of cerebral palsy. BMC Neurol 2014;14:203.

45 Morgan C, Novak I, Dale RC, et al. Single blind randomised controlled trial of GAME (Goals - Activity - Motor Enrichment) in infants at high risk of cerebral palsy. Res Dev Disabil 2016;55:256-67.

46 Sgandurra G, Lorentzen J, Inguaggiato E, et al. A randomized clinical trial in preterm infants on the effects of a home-based early intervention with the 'CareToy System'. PLoS One 2017; 12:e0173521.

47 Bilde PE, Kliim-Due M, Rasmussen B, et al. Individualized, homebased interactive training of cerebral palsy children delivered through the Internet. BMC Neurol 2011;11:32.

48 Campoy C, Escolano-Margarit MV, Anjos T, et al. Omega 3 fatty acids on child growth, visual acuity and neurodevelopment. Br J Nutr 2012;107 Suppl 2:S85-106.

49 Ochi E, Tsuchiya Y. Eicosapentaenoic acid (EPA) and docosahexaenoic acid (DHA) in muscle damage and function. Nutrients 2018;10. doi:10.3390/nu10050552. [Epub ahead of print: 2904 2018].

50 Jasani B, Simmer K, Patole SK, et al. Long chain polyunsaturated fatty acid supplementation in infants born at term. Cochrane Database Syst Rev 2017;3:Cd000376.

51 Lapillonne A, Moltu SJ. Long-Chain polyunsaturated fatty acids and clinical outcomes of preterm infants. Ann Nutr Metab 2016;69 Suppl 1:35-44.

52 Ogaz-González R, Mérida-Ortega Ángel, Torres-Sánchez $\mathrm{L}$, et al. Maternal dietary intake of polyunsaturated fatty acids modifies association between prenatal DDT exposure and child neurodevelopment: a cohort study. Environ Pollut 2018;238:698-705.

53 Starkey JD. Triennial Growth Symposium--A role for vitamin D in skeletal muscle development and growth. J Anim Sci 2014;92:887-92.

54 Koundourakis NE, Avgoustinaki PD, Malliaraki N, et al. Muscular effects of vitamin $\mathrm{D}$ in young athletes and non-athletes and in the elderly. Hormones 2016;15:471-88.

55 Ali A, Cui X, Eyles D. Developmental vitamin D deficiency and autism: putative pathogenic mechanisms. J Steroid Biochem Mol Biol 2018;175:108-18.

56 Zinglersen $\mathrm{AH}$, Halsteen MB, Kjaer M, et al. Can electrical stimulation enhance effects of a functional training program in hospitalized geriatric patients? Exp Gerontol 2018;106:101-8.

57 Kern H, Hofer C, Loefler S, et al. Atrophy, ultra-structural disorders, severe atrophy and degeneration of denervated human muscle in $\mathrm{SCl}$ and aging. Implications for their recovery by functional electrical stimulation, updated 2017. Neurol Res 2017;39:660-6.

58 Sgandurra G, Beani E, Giampietri M, et al. Early intervention at home in infants with congenital brain lesion with CareToy revised: a RCT protocol. BMC Pediatr 2018;18:295.

59 Novak I, Morgan C, Adde L, et al. Early, accurate diagnosis and early intervention in cerebral palsy: advances in diagnosis and treatment. JAMA Pediatr 2017;171:897-907.

60 Herskind A, Willerslev-Olsen M, Ritterband-Rosenbaum A, et al. Intensive gait training in toddlers with cerebral palsy: a pilot study. Journal of Advances in Pediatric Research 2016;3:1-10.

61 Nielsen JB, Willerslev-Olsen M, Christiansen L, et al. Science-Based neurorehabilitation: recommendations for neurorehabilitation from basic science. J Mot Behav 2015;47:7-17.

62 Christensen MS, Grünbaum T. Sense of agency for movements. Conscious Cogn 2018;65:27-47.

63 Rochat P, Morgan R. Spatial determinants in the perception of selfproduced leg movements in 3- to 5-month-old infants. Dev Psychol 1995;31:626-36.

64 Sgandurra G, Bartalena L, Cecchi F, et al. A pilot study on early home-based intervention through an intelligent baby gym (CareToy) in preterm infants. Res Dev Disabil 2016;53-54:32-42.

65 Karabay İlkay, Öztürk GT, Malas Fevziye Ünsal, et al. Short-Term effects of neuromuscular electrical stimulation on muscle architecture of the tibialis anterior and gastrocnemius in children with cerebral palsy: preliminary results of a prospective controlled study. $A m \mathrm{~J}$ Phys Med Rehabil 2015;94:728-33.

66 Barber L, Barrett R, Lichtwark G. Validation of a freehand 3D ultrasound system for morphological measures of the medial gastrocnemius muscle. J Biomech 2009;42:1313-9.

67 Barber L, Barrett R, Lichtwark G. Validity and reliability of a simple ultrasound approach to measure medial gastrocnemius muscle length. J Anat 2011;218:637-42.

68 Herskind A, Greisen G, Nielsen JB. Early identification and intervention in cerebral palsy. Dev Med Child Neurol 2015;57:29-36.

69 Khamis A, Novak I, Morgan C, et al. Motor learning feeding interventions for infants at risk of cerebral palsy: a systematic review. Dysphagia 2020;35:1-17.

70 Spittle AJ, Morgan C, Olsen JE, et al. Early diagnosis and treatment of cerebral palsy in children with a history of preterm birth. Clin Perinatol 2018;45:409-20. 